\title{
REFLECTION AND WEAKLY COLLECTIONWISE HAUSDORFF SPACES
}

\author{
TIM LABERGE AND AVNER LANDVER
}

(Communicated by Franklin D. Tall)

\begin{abstract}
We show that $\square(\theta)$ implies that there is a first countable $<\theta$ collectionwise Hausdorff space that is not weakly $\theta$-collectionwise Hausdorff. We also show that in the model obtained by Levy collapsing a weakly compact (supercompact) cardinal to $\omega_{2}$, first countable $\aleph_{1}$-collectionwise Hausdorff spaces are weakly $\aleph_{2}$-collectionwise Hausdorff (weakly collectionwise Hausdorff). In the last section we show that assuming $E_{\theta}^{\omega}$, a certain $\theta$-family of integer-valued functions exists and that in the model obtained by Levy collapsing a supercompact cardinal to $\omega_{2}$, these families do not exist.
\end{abstract}

\section{INTRODUCTION}

Reflection is a central theme in modern set-theoretic topology. As Alan Dow points out in [Do], we often prove theorems when some type of reflection principle holds, and we build counterexamples when reflection fails. This paper contains both types of results, on questions related to the failure of collectionwise Hausdorff.

We say that a subset $A$ of a topological space $X$ can be separated if there is a collection $\left\{U_{x}: x \in A\right\}$ of disjoint open sets with $x \in U_{x}$ for every $x \in A$. A space $X$ is $<\theta$-collectionwise Hausdorff $(<\theta$-cwH) if every closed discrete subset of size $<\theta$ can be separated. A space $X$ is $\leq \theta$-collectionwise Hausdorff ( $\leq \theta$-cwH) if every closed discrete subset of size $\leq \theta$ can be separated. $X$ is collectionwise Hausdorff ( $\mathrm{cwH}$ ) if it is $\leq \theta-\mathrm{cwH}$ for every cardinal $\theta$.

In particular, we are concerned with Fleissner's questions:

Question 1. Is there a ZFC example of a first countable $<\aleph_{2}-c w H$ space that is $n o t \leq \aleph_{2}-c w H$ ?

Question 2. Is there a ZFC example of a first countable $<\aleph_{2}-c w H$ space that is not $c w H$ ?

Fleissner asks for ZFC examples because he showed [F] that $E_{\omega_{2}}^{\omega}$ (a nonreflecting stationary subset of $\left.\left\{\alpha<\omega_{2}: c f(\alpha)=\omega\right\}\right)$ can be used to construct an example of a first countable $<\aleph_{2}-\mathrm{cwH}$ space that is not $\leq \aleph_{2}-\mathrm{cwH}$. Since the

Received by the editors December 4, 1992.

1991 Mathematics Subject Classification. Primary 54D15; Secondary 54A35, 04A72.

Key words and phrases. Reflection, weakly collectionwise Hausdorff, Levy forcing, Mitchell forcing, fans. 
failure of $E_{\omega_{2}}^{\omega}$ is equiconsistent with the existence of a Mahlo cardinal [De, HS], large cardinals are required for a negative answer to Question 1. In fact, we will see that by a result of Todorcevic [To2], at least a weakly compact cardinal will be needed to get a negative answer to Question 1. Shelah has shown [S] that, in the model obtained by Levy collapsing a weakly compact cardinal to $\omega_{2}$, first countable $<\aleph_{2}-\mathrm{cwH}$ spaces that are locally of size at most $\aleph_{1}$ are $\leq \aleph_{2}-\mathrm{cwH}$, and that in the model obtained by collapsing a supercompact cardinal, such spaces are $\mathrm{cwH}$. For a more complete history of the general problem of reflecting the failure of $\mathrm{cwH}$, see [W, FS]. We thank Bill Fleissner for his valuable input and Gary Gruenhage for letting us use the handwritten notes [GT].

\section{SQUARES OF FANS AND FIRST COUNTABLE COLLECTIONWISE HAUSDORFF SPACES}

Recently, Gruenhage and Tamano [GT] have discovered a connection between Fleissner's questions and the problem of determining the tightness of the squares of certain fans. Before we discuss this connection, we make some definitions.

The fan $F_{\theta, \omega}$ is the quotient space obtained by identifying the nonisolated points of $\theta$-many copies of the convergent $\omega$-sequence. To be precise, $F_{\theta, \omega}=$ $\{*\} \cup(\theta \times \omega)$, topologized so that points of $\theta \times \omega$ are isolated and so that an open base at $*$ is the family of all

$$
B_{f}=\{*\} \cup\{(\alpha, n): n \geq f(\alpha)\} \quad\left(f \in{ }^{\theta} \omega\right) .
$$

When working with the square of $F_{\theta, \omega}$, one can always replace two functions $f_{1}, f_{2} \in{ }^{\theta} \omega$ with $g=\max \left(f_{1}, f_{2}\right)$, so we use the family of all

$$
V_{g}=B_{g} \times B_{g} \quad\left(g \in{ }^{\theta} \omega\right)
$$

as an open base for $(*, *)$ in $F_{\theta, \omega} \times F_{\theta, \omega}$.

We say that a set $S \subseteq F_{\theta, \omega} \times F_{\theta, \omega}$ is $\theta$-good if $(*, *) \in \bar{S}$, but for all $T \in[S]^{<\theta},(*, *) \notin \bar{T}$. Thus, "there is a $\theta$-good set in $F_{\theta, \omega} \times F_{\theta, \omega}$ " means that the tightness of $F_{\theta, \omega} \times F_{\theta, \omega}$ is $\theta$ and that this tightness is actually attained.

Finally, we say that a set $H \in[\omega \times \omega]^{<\omega}$ is closed downward (c.d.w.) if whenever $(n, m) \in H$, then $n^{\prime} \leq n$ and $m^{\prime} \leq m$ implies $\left(n^{\prime}, m^{\prime}\right) \in H$.

The Gruenhage-Tamano result is the equivalence $(1) \Longleftrightarrow(2)$ in Theorem 1. We have added the combinatorial equivalence (3) and the superficially stronger topological characterization (4).

Theorem 1. Let $\theta$ be an uncountable cardinal. TFAE:

(1) There exists a $\theta$-good subset of $F_{\theta, \omega} \times F_{\theta, \omega}$.

(2) There exists a space $X$ that is first countable, $<\theta-c w H$ and not $\leq \theta-c w H$.

(3) There exists $\mathscr{H}=\left\{H_{\alpha \beta}: \alpha<\beta<\theta\right\} \subseteq[\omega \times \omega]^{<\omega}$ with each $H_{\alpha \beta}$ c.d.w. such that:

(a) for every $A \in[\theta]^{<\theta}$ there is a function $f: \theta \rightarrow \omega$ such that for all $\alpha<\beta$ in $A,(f(\alpha), f(\beta)) \notin H_{\alpha \beta}$

(b) for every $f: \theta \rightarrow \omega$ there are $\alpha<\beta<\theta$ such that $(f(\alpha), f(\beta)) \in H_{\alpha \beta}$.

(4) There exists a space $X$ that is first countable, zero-dimensional, $<\theta-c w H$ and not $\leq \theta-c w H$.

In order to put Theorem 1 in perspective, we need the definition of a difficultto-deny combinatorial principle. For $\theta$ an uncountable regular cardinal, $\square(\theta)$ 
is the assertion that there is a family $\left\{C_{\alpha}: \alpha<\theta\right\}$ satisfying the following conditions:

(i) $C_{\alpha} \subset \alpha$ is a club subset of $\alpha$.

(ii) If $\alpha$ is a limit point of $C_{\beta}$, then $C_{\alpha}=C_{\beta} \cap \alpha$.

(iii) There is no club $C \subset \theta$ such that for every limit point $\alpha$ of $C$, $C_{\alpha}=C \cap \alpha$.

$\square(\theta)$ is true for every regular $\theta$ which is not weakly compact in $L$ (see [Tol]). Todorcevic showed (see [To2, Be]) that $\square(\theta)$ can be used to construct a $\theta$-good set. Also, combining Theorem 1 with Fleissner's construction of a first countable, $<\theta$-cwH, not $\leq \theta$-cwH space from $E_{\theta}^{\omega}$, we see that $E_{\theta}^{\omega}$ can be used to construct a $\theta$-good set.

Because the proof of Theorem 1 is essentially the same as the proof of Theorem 2, we only prove Theorem 2 . Before the statement of the theorem we define a weakening of collectionwise Hausdorff introduced by Tall [Ta1]. We say that a subset $A$ of a space $X$ is weakly separated if it has a subset of size $|A|$ that is separated. $X$ is weakly $\theta-c w H$ if every closed discrete subset of size $\theta$ is weakly separated. $X$ is weakly $c w H$ if it is weakly $\theta$-cwH for every cardinal $\theta$.

Theorem 2. Let $\kappa \leq \theta$ be uncountable cardinals. TFAE:

(1) There exists a space $X$ that is first countable, $<\kappa-c w H$, and not weakly $\theta-c w H$.

(2) There exists $\mathscr{H}=\left\{H_{\alpha \beta}: \alpha<\beta<\theta\right\} \subset[\omega \times \omega]^{<\omega}$, with each $H_{\alpha \beta}$ c.d.w., such that:

(a) for every $A \in[\theta]^{<\kappa}$ there is a function $f: \theta \rightarrow \omega$ such that for all $\alpha<\beta$ in $A,(f(\alpha), f(\beta)) \notin H_{\alpha \beta}$;

(b) for every $B \in[\theta]^{\theta}$ and every $f: \theta \rightarrow \omega$ there are $\alpha<\beta$ in $B$ such that $(f(\alpha), f(\beta)) \in H_{\alpha \beta}$.

(3) There exists a space $X$ that is first countable, zero-dimensional, $<\kappa-c w H$, and not weakly $\theta$-cwH.

Proof. (1) $\Rightarrow(2)$ : Let $\theta \subset X$ be a subset which is not weakly separated. For every $\alpha \in \theta$ let $\left\{U_{n}(\alpha): n \in \omega\right\}$ be a decreasing neighbourhood base at $\alpha$. Let

$$
S=\left\{(\langle\alpha, n\rangle,\langle\beta, m\rangle): U_{n}(\alpha) \cap U_{m}(\beta) \neq \varnothing\right\} .
$$

$S$ is a subset of $F_{\theta, \omega} \times F_{\theta, \omega}$. For every $B \subset \theta$, let $S_{B}=\{(\langle\alpha, n\rangle,\langle\beta, m\rangle) \in$ $S: \alpha, \beta \in B\}$. We claim that $S$ satisfies the following two conditions

(i) For every $A \in[\theta]^{<\kappa},(*, *) \notin \bar{S}_{A}$.

(ii) For every $B \in[\theta]^{\theta},(*, *) \in \bar{S}_{B}$.

To show (i), let $A \in[\theta]^{<\kappa}$. Let $f: A \rightarrow \omega$ be such that $\left\{U_{f(\alpha)}(\alpha): \alpha \in A\right\}$ is a separation of $A$ in $X$. Let $V_{f}$ be the open neighbourhood of $(*, *)$ in $F_{\theta, \omega} \times F_{\theta, \omega}$ that is determined by $f$. It is easy to check that $V_{f} \cap S_{A}=\varnothing$. For (ii), let $B \in[\theta]^{\theta}$. Let $f: \theta \rightarrow \omega$, and let $V_{f}$ be as above. Now, $f \backslash B$ is not a code for a separation of $B$ in $X$; therefore, there are $\alpha<\beta$ in $B$ such that $U_{f(\alpha)}(\alpha) \cap U_{f(\beta)}(\beta) \neq \varnothing$. But then $(\langle\alpha, f(\alpha)\rangle,\langle\beta, f(\beta)\rangle) \in S_{B} \cap V_{f}$.

Using the fact that $(*, *)$ is not in the closure of any countable subset of $S$, it is not hard to verify the following facts. 
Fact 1. For every $\alpha<\theta$ there is $h(\alpha) \in \omega$ such that for every $n>h(\alpha)$ and for every $\beta<\theta$ the set $\{m \in \omega:(\langle\alpha, n\rangle,\langle\beta, m\rangle) \in S\}$ is finite.

Fact 2. For every $\beta<\theta$ there is $g(\beta) \in \omega$ such that for every $m>g(\beta)$ and for every $\alpha<\theta$ the set $\{n \in \omega:(\langle\alpha, n\rangle,\langle\beta, m\rangle) \in S\}$ is finite.

Let $T=S \cap\left(B_{h} \times B_{g}\right)$. Clearly, $T$ satisfies (i) and (ii). Now for every $\alpha<\beta<\theta$ define

$$
H_{\alpha \beta}^{\prime}=\{(n, m) \in \omega \times \omega:(\langle\alpha, n\rangle,\langle\beta, m\rangle) \in T\} .
$$

Let $H_{\alpha \beta}$ be the downward closure of $H_{\alpha \beta}^{\prime}$. Let us show that the $H_{\alpha \beta}$ 's are finite. It is enough to show that the $H_{\alpha \beta}^{\prime}$ 's are finite. Assume that this is false, and let $\alpha<\beta<\theta$ be such that $H_{\alpha \beta}^{\prime}$ is infinite. It follows that for every $k \in \omega$ there are $n, m>k$ such that $(n, m) \in H_{\alpha \beta}^{\prime}$. This implies that, $(*, *)$ is in the closure of $\left\{(\langle\alpha, n\rangle,\langle\beta, m\rangle):(n, m) \in H_{\alpha \beta}^{\prime}\right\}$; therefore $(*, *)$ is in the closure of a countable subset of $T$, a contradiction.

Finally, it is not hard to see that $\left\{H_{\alpha \beta}: \alpha<\beta<\theta\right\}$ satisfies (a) and (b) of (2) precisely because $T$ satisfies (i) and (ii).

$(2) \Rightarrow(3):$ As in [GT], first let $I=\{(\langle\alpha, n\rangle,\langle\beta, m\rangle): \alpha<\beta<\theta \wedge(n, m) \in$ $\left.H_{\alpha \beta}\right\}$. Then let $X=I \cup \theta$. Points in $I$ are isolated, and for every $\gamma \in \theta$ a base at $\gamma$ is given by

$$
\begin{aligned}
U_{k}(\gamma)=\{\gamma\} \cup\{(\langle\alpha, n\rangle,\langle\beta, m\rangle) & \in I: \\
(\alpha=\gamma \wedge n \geq k) & \vee(\beta=\gamma \wedge m \geq k)\} \quad(k \in \omega) .
\end{aligned}
$$

Clearly, $X$ is first countable. To see that $X$ is $<\kappa$-cwH let $A \in[\theta]^{<\kappa}$. Let $f: \theta \rightarrow \omega$ be given by (2)(a). Then check that $\left\{U_{f(\alpha)}(\alpha): \alpha \in A\right\}$ is a separation of $A$. To see that $X$ is not weakly $\theta$-cwH let $B \in[\theta]^{\theta}$ and $f: \theta \rightarrow \omega$. By (2)(b), there are $\alpha<\beta$ in $B$ such that $(f(\alpha), f(\beta)) \in H_{\alpha \beta}$. So

$$
(\langle\alpha, f(\alpha)\rangle,\langle\beta, f(\beta)\rangle) \in U_{f(\alpha)}(\alpha) \cap U_{f(\beta)}(\beta) ;
$$

therefore, $f$ does not code a separation of $B$.

Finally, the finiteness of the $H_{\alpha \beta}$ 's implies that each $U_{k}(\alpha)$ is clopen, and therefore $X$ is zero-dimensional.

(3) $\Rightarrow(1)$ : Trivial.

\section{INDEPENDENCE RESULTS}

In this section, we first show that $\square(\theta)$ implies that there is a first countable, $<\theta$-cwH space that is not weakly $\theta-\mathrm{cwH}$. We then demonstrate the consistency of "first countable $<\aleph_{2}$-cwH spaces are weakly $\aleph_{2}-\mathrm{cwH}$ (weakly cwH)", assuming the consistency of "there is a weakly compact (supercompact) cardinal".

Since $\square(\theta)$ is true unless $\theta$ is weakly compact in $L$, this gives that "first countable $<\aleph_{2}$-cwH spaces are weakly $\aleph_{2}$-cwH" is equiconsistent with "there is a weakly compact cardinal" and that the consistency of "first countable $<\aleph_{2}$ cwH spaces are weakly cwH" implies the existence of an inner model with many measurable cardinals $[\mathrm{KM}]$.

Theorem 3. Let $\theta$ be a regular uncountable cardinal, and suppose that $\square(\theta)$ holds. Then there is a zero-dimensional, first countable, $<\theta-c w H$ space that is not weakly $\theta-c w H$. 
Proof. In [Be], Todorcevic constructs, from the assumption of $\square(\theta)$, a function $\rho_{2}$ that maps pairs $\alpha<\beta<\theta$ into $\omega$. In $\S 4$ we will study integer-valued families of functions, so to keep our notation consistent, we define for each $\beta<\theta$ a function $h_{\beta}: \beta \rightarrow \omega$ by

$$
h_{\beta}(\alpha)=\rho_{2}(\alpha, \beta) \quad(\alpha<\beta) .
$$

By the properties of $\rho_{2}$ cited in [Be], the family $\left\{h_{\beta}: \beta<\theta\right\}$ satisfies

(1) For all $\alpha<\beta<\theta$, there is an $n_{\alpha \beta} \in \omega$ such that for all $\xi<\alpha$, $h_{\alpha}(\xi)<h_{\beta}(\xi)+n_{\alpha \beta}$. (This is monotonicity [DW].)

(2) For all $B \in[\theta]^{\theta}$ and all $n \in \omega$, there exist $\alpha<\beta$ in $B$ such that $h_{\beta}(\alpha)>n$.

For every $\alpha<\beta<\theta$, define $H_{\alpha \beta}=\left\{(n, m): n+m \leq h_{\beta}(\alpha)\right\}$. Let us show that $\mathscr{H}=\left\{H_{\alpha \beta}: \alpha<\beta<\theta\right\}$ satisfies (2) of Theorem 2 with $\kappa=\theta$. by

For (2)(a) let $A \in[\theta]^{<\kappa}$. Let $\gamma<\theta$ be a bound for $A$, and define $f: \theta \rightarrow \omega$

$$
f(\alpha)= \begin{cases}h_{\gamma}(\alpha)+n_{\alpha \gamma} & \text { if } \alpha<\gamma, \\ 0 & \text { otherwise. }\end{cases}
$$

Now let $\alpha<\beta$ in $A$, then $f(\alpha)+f(\beta) \geq h_{\gamma}(\alpha)+n_{\beta \gamma}>h_{\beta}(\alpha)$. Therefore, $(f(\alpha), f(\beta)) \notin H_{\alpha \beta}$.

For (2)(b) let $B \in[\theta]^{\theta}$ and $f: \theta \rightarrow \omega$. Then there exist $B^{\prime} \in[B]^{\theta}$ and $n \in \omega$ such that for every $\alpha \in B^{\prime}, f(\alpha)=n$. But then there are $\alpha<\beta$ in $B^{\prime}$ such that $h_{\beta}(\alpha)>2 n$. Hence $f(\alpha)+f(\beta)=2 n<h_{\beta}(\alpha)$, so $(f(\alpha), f(\beta)) \in$ $H_{\alpha \beta}$.

Since the $h_{\beta}$ 's also satisfy

(3) whenever $A, B \in[\theta]^{\theta}$ and $n \in \omega$, there are $\alpha<\beta$ with $\alpha \in A$ and $\beta \in B$ such that $h_{\beta}(\alpha)>n$,

it follows that the space constructed above is badly nonnormal.

In [Ta2], Tall asks if $G C H$ is consistent with the existence of a first countable, weakly $\aleph_{1}$-cwH space that is not weakly $\aleph_{2}-\mathrm{cwH}$. He asked for a consistent with $G C H$ example because Daniels has constructed [D] such a space from $M A+\neg C H$. Our example answers Tall's question positively, is $\left\langle\aleph_{2}-\mathrm{cwH}\right.$, and exists unless there is a weakly compact cardinal in $L$.

The next two theorems will use the method of forcing + reflection. Good references for this technique are [DTW1] and [DTW2]. We first prove a preservation lemma, whose proof is motivated by Lemma 3.13 in [Be].

Main Lemma. Assume that $\mathbb{P}$ is an $\omega_{1}$-closed partial order and that $\theta \geq \omega_{2}$ is a regular cardinal. Assume that $\mathscr{H}=\left\{H_{\alpha \beta}: \alpha<\beta<\theta\right\} \subset[\omega \times \omega]^{<\omega}$ are c.d.w., and the following condition from Theorem 2 holds:

(b) For every $B \in[\theta]^{\theta}$ and every $f: \theta \rightarrow \omega$ there are $\alpha<\beta$ in $B$ such that $(f(\alpha), f(\beta)) \in H_{\alpha \beta}$.

Assume that $G$ is a $\mathbb{P}$-generic filter over $V$. Then in $V[G]$, for every $f: \theta \rightarrow \omega$ there are $\alpha<\beta<\theta$ such that $(f(\alpha), f(\beta)) \in H_{\alpha \beta}$.

Proof. Assume that the lemma is false. Then there is $p_{0} \in \mathbb{P}$ and a $\mathbb{P}$-name $\dot{g}$ such that

$$
p_{0} \Vdash “ \dot{g}: \theta \rightarrow \omega \wedge(\forall \alpha<\beta<\theta)(\dot{g}(\alpha), \dot{g}(\beta)) \notin H_{\alpha \beta} ” .
$$


Let $M$ be an elementary substructure of some $H(\lambda)$ ( $\lambda$ large enough) with $\theta, \mathscr{H}, \mathbb{P}, p_{0}, \dot{g}$, and $\vdash_{\mathbb{P}}$ in $M$ and $M \cap \theta=\beta<\theta$.

If possible, choose $p_{1} \leq p_{0}$ and $\alpha_{0}<\beta$ such that $p_{1} \in M$ and $p_{1} \Vdash$ “ $\left(\dot{g}\left(\alpha_{0}\right), 0\right) \in H_{\alpha_{0} \beta}$ ". Note that this would imply that $p_{1} \Vdash$ " $\dot{g}(\beta)>0$ ".

Similarly construct $p_{0} \geq p_{1} \geq \cdots \geq p_{n+1} \cdots$ such that $p_{n+1} \in M$ and $\alpha_{n}<\beta$ with

$$
p_{n+1} \Vdash “\left(\dot{g}\left(\alpha_{n}\right), n\right) \in H_{\alpha_{n} \beta} " .
$$

In particular, this would imply that $p_{n+1} \Vdash$ " $\dot{g}(\beta)>n$ ". This process must stop at a finite stage since otherwise one could choose $q$ with $q \leq p_{n}$ for every $n \in \omega$ and get that for every $n \in \omega, q \Vdash$ " $\dot{g}(\beta)>n$ ", which is impossible.

So, there is $\bar{n} \in \omega$ such that for every $q \leq p_{\bar{n}}$ and for every $\alpha<\beta$, if $q \in M$, then

$$
q \nVdash “(\dot{g}(\alpha), \bar{n}) \in H_{\alpha \beta} \text { ”. }
$$

Claim 1. For every $\alpha<\beta, p_{\bar{n}} \Vdash$ “ $(\dot{g}(\alpha), \bar{n}) \notin H_{\alpha \beta}$ ”.

Proof. Assume not. Then there is $q \leq p_{n}$ and $\alpha<\beta$ such that $q \Vdash$ “( $\dot{g}(\alpha), \bar{n}) \in H_{\alpha \beta}$ ". Fix such $\alpha<\beta$ and the corresponding $H_{\alpha \beta}$. Then $H(\lambda) \models$ $\left(\exists q \leq p_{n}\right) q \Vdash$ “ $(\dot{g}(\alpha), \bar{n}) \in H_{\alpha \beta}$ ". But $p_{\bar{n}}, \Vdash_{\mathbb{P}}, \dot{g}, \alpha$, and $H_{\alpha \beta}$ are all in $M$; therefore,

$$
M \models \exists q \leq p_{\bar{n}}\left(q \Vdash \text { “ }(\dot{g}(\alpha), \bar{n}) \in H_{\alpha \beta} "\right),
$$

which is a contradiction.

Let $B=\left\{\beta \in \theta: \forall \alpha<\beta\left(p_{n} \Vdash\right.\right.$ “ ( $\left.\dot{g}(\alpha), \bar{n}\right) \notin H_{\alpha \beta}$ ”) . By elementarity of $M,|B|=\theta$.

Now, for every $\alpha \in \theta$ let $q_{\alpha} \leq p_{n}$ and $m_{\alpha} \in \omega$ be such that $q_{\alpha} \Vdash$ " “ $\dot{g}(\alpha)=$ $m_{\alpha}$. In $V$, define a function $f: \theta \rightarrow \omega$ by

$$
f(\alpha)=m_{\alpha}+\bar{n} \quad(\alpha \in \theta) .
$$

The following claim will give us the desired contradiction.

Claim 2. For every $\beta \in B$ and every $\alpha<\beta,(f(\alpha), f(\beta)) \notin H_{\alpha \beta}$.

Proof. Fix $\beta \in B$ and $\alpha<\beta$. By definition, $f(\alpha) \geq m_{\alpha}$ and $f(\beta) \geq \bar{n}$, therefore it is enough to show that $\left(m_{\alpha}, \bar{n}\right) \notin H_{\alpha \beta}$. But $p_{n} \Vdash$ “ $(\dot{g}(\alpha), \bar{n}) \notin$ $H_{\alpha \beta}$ ", and $q_{\alpha} \leq p_{n}$, and $q_{\alpha} \Vdash$ " $\dot{g}(\alpha)=m_{\alpha}$ ". Therefore $q_{\alpha} \Vdash$ " $\left(m_{\alpha}, \bar{n}\right) \notin H_{\alpha \beta}$ ", and this implies that indeed $\left(m_{\alpha}, \bar{n}\right) \notin H_{\alpha \beta}$.

Let $\kappa$ be strongly inaccessible and let $\mathbb{P}=\mathbb{P}_{\kappa}$ be the Levy collapse of $\kappa$ to $\omega_{2}$ with countable conditions (for a definition and proofs of the facts below, see $[\mathrm{K}])$. Let $G$ be a $\mathbb{P}$-generic filter. We will use the following facts about $\mathbb{P}: \mathbb{P}$ is an $\omega_{1}$-closed partial order. $\mathbb{P}$ is $\kappa$-c.c. and $\kappa=\omega_{2}$ in $V[G]$. For every $\lambda<\kappa, \mathbb{P}$ can be factored as $\mathbb{P}=\mathbb{P}_{\lambda} \times \mathbb{P}^{\lambda}, \mathbb{P} \cap V_{\lambda}=\mathbb{P}_{\lambda}$ and in $V\left[G_{\lambda}\right]$, $\mathbb{P}^{\lambda}$ is forcing equivalent to $\mathbb{P}$ (in particular, it is $\omega_{1}$-closed). For a definition and discussion of weakly compact cardinals, see $[\mathrm{K}, \mathrm{KM}]$. The following type of proof is well known [Ba, Mi]; see also [DTW2].

Theorem 4. Assume that $\kappa$ is a weakly compact cardinal. Let $\mathbb{P}$ be the Levy collapse of $\kappa$ to $\omega_{2}$ with countable conditions, and let $G$ be a $\mathbb{P}$-generic filter over $V$. Then in $V[G]$, every first countable $\left\langle\aleph_{2}-c w H\right.$ space is weakly $\aleph_{2}-c w H$. Proof. By way of contradiction, suppose that in $V[G]$ there is a first countable $<\aleph_{2}$-cwH space that is not weakly $\aleph_{2}$-cwH. In $V[G], \omega_{2}=\kappa$; therefore, there is $\mathscr{H}=\left\{H_{\alpha \beta}: \alpha<\beta<\kappa\right\}$ that satisfies (2) of Theorem 2 with $\kappa=\theta$. This 
fact must be forced, and the forcing statement is $\Pi_{1}^{1}$ over $\left(V_{\kappa}, \epsilon\right)$ (with few extra parameters). Using the facts that $\kappa$ is weakly compact ( $\Pi_{1}^{1}$-reflection) and that $\mathbb{P}$ is $\kappa$-c.c., we find an inaccessible $\lambda<\kappa$. such that in $V\left[G_{\lambda}\right],\left\{H_{\alpha \beta}\right.$ : $\alpha<\beta<\lambda$ \} satisfies (2) of Theorem 2 (with $\lambda$ playing the role of both $\kappa$ and $\theta)$.

Now $V\left[G_{\lambda}\right] \models \mathbb{P}^{\lambda}$ is $\omega_{1}$-closed. Therefore, by the Main Lemma, for every function $f: \lambda \rightarrow \omega$ in $V[G]$, there are $\alpha<\beta<\lambda$ such that $(f(\alpha), f(\beta)) \in$ $H_{\alpha \beta}$. But $\lambda<\kappa$; therefore, this contradicts the fact that (2)(a) of Theorem 2 holds for $\mathscr{H}$ in $V[G]$.

For a definition and discussion of supercompact cardinals see [KM, J, DTW1].

Theorem 5. Assume that $\kappa$ is a supercompact cardinal and that the $G C H$ holds above $\kappa$. Let $\mathbb{P}$ be the Levy collapse of $\kappa$ to $\omega_{2}$ with countable conditions, and let $G$ be a $\mathbb{P}$-generic filter over $V$. Then in $V[G]$ every first countable $<\aleph_{2}-c w H$ space is weakly $\mathrm{cwH}$.

Proof. We have to show that in $V[G]$, if $\theta \geq \omega_{2}$ is a cardinal and $X$ is a first countable, $\left\langle\aleph_{2}-\mathrm{cwH}\right.$ space, then $X$ is weakly $\theta$-cwH. It is a consequence of a result of Watson [W] that if $\theta$ is a singular strong limit cardinal and $X$ is first countable and weakly $<\theta$-cwH, then $X$ is weakly $\theta$-cwH. Therefore, we may assume that $\theta$ is regular. Since $\kappa=\omega_{2}$ in $V[G]$, we have $\theta \geq \kappa$. The plan is to show that (2) of Theorem 2, with $\kappa=\omega_{2}$, fails in $V[G]$.

Let $j: V \rightarrow M$ be an elementary embedding such that:

(i) $j(\alpha)=\alpha$, for all $\alpha<\kappa$,

(ii) $j(\kappa)>\theta$,

(iii) ${ }^{\theta} M \cap V \subset M$.

Now, $\mathbb{P} \subset V_{\kappa}$ and $j \mid V_{\kappa}$ is the identity map; therefore, $\mathbb{P} \subset M$ and by (iii) $\mathbb{P} \in M$. So $M[G]$ makes sense. In $M$ (and also in $V$ ), $j(\mathbb{P})$ is the Levy collapse of $j(\kappa)$ to $\omega_{2}$. In the usual way $j(\mathbb{P})$ can be factored, so $j(\mathbb{P})=j(\mathbb{P})_{\kappa} \times j(\mathbb{P})^{\kappa}$. But $j(\mathbb{P})_{\kappa}=\mathbb{P}$, so $j(\mathbb{P})=\mathbb{P} \times j(\mathbb{P})^{\kappa}$. Moreover,

$$
M[G] \models j(\mathbb{P})^{\kappa} \text { is an } \omega_{1} \text {-closed partial order. }
$$

Let $I$ be a $j(\mathbb{P})^{\kappa}$-generic filter over $V[G] . \quad I$ is also $j(\mathbb{P})^{\kappa}$-generic over $M[G]$, and there is a filter $K \subset j(\mathbb{P})$ such that $M[G][I]=M[K]$ and $V[G][I]=$ $V[K]$. Using the fact that for every $p \in \mathbb{P}, j(p)=p$, we can define, in $V[K]$,

$$
j^{*}: V[G] \rightarrow M[K]
$$

that extends $j$ and show that it is an elementary embedding (see [KM, §25]).

Let $\mathscr{H} \in V[G]$ with $\mathscr{H}=\left\{H_{\alpha \beta}: \alpha<\beta<\theta\right\} \subset[\omega \times \omega]^{<\omega}$ c.d.w., and assume that the following condition from Theorem 2 holds in $V[G]$ :

(b) For every $B \in[\theta]^{\theta}$ and every $f: \theta \rightarrow \omega$ there are $\alpha<\beta$ in $B$ such that $(f(\alpha), f(\beta)) \in H_{\alpha \beta}$.

We will show that (a) from Theorem 2 (with $\kappa=\omega_{2}$ ) is false for $\mathscr{H}$ in $V[G]$.

$\mathscr{H}$ has a name of size $\theta$ in $V$ that is a subset of $M$; therefore, by (iii) this name is in $M$. Hence, $\mathscr{H} \in M[G]$ and it is clear that condition, (b) holds for $\mathscr{H}$ in $M[G]$ as well. Notice that formally $\mathscr{H}$ is a function given by $\mathscr{H}=\left\{\left(\langle\alpha, \beta\rangle, H_{\alpha \beta}\right): \alpha<\beta<\theta\right\}$. Let $\mathscr{H}^{\prime}=j^{*}(\mathscr{H}) \in M[K]$. Then $\mathscr{H}^{\prime}=\left\{\left\langle\langle\gamma, \delta\rangle, H_{\gamma \delta}^{\prime}\right\rangle: \gamma<\delta<j(\theta)\right\}$, where each $H_{\gamma \delta}^{\prime}$ is a c.d.w. subset of 
$[\omega \times \omega]^{<\omega}$. Notice that for every $\alpha<\beta<\theta, H_{\alpha \beta}=j\left(H_{\alpha \beta}\right)=H_{j(\alpha) j(\beta)}^{\prime}$. Let $A=j^{\prime \prime} \theta$, then $j^{\prime \prime} \mathscr{H}=j^{* \prime \prime} \mathscr{C}=\left\{\left(\langle j(\alpha), j(\beta)\rangle, H_{\alpha \beta}\right): \alpha<\beta<\theta\right\}=$ $\left\{\left(\langle\gamma, \delta\rangle, H_{\gamma \delta}^{\prime}\right):(\gamma<\delta) \wedge(\gamma, \delta \in A)\right\} \subset \mathscr{H}^{\prime}$. The map $j \mid \mathscr{H}$ is a subset of $M$ of size $\theta$, but it is not in $V$; therefore, we cannot conclude that it is in $M$. Fortunately, ${ }^{\theta} M[G] \cap V[G] \subset M[G]$ (see [J, p. 463]); therefore, $j\lceil\mathscr{H} \in M[G] \subset M[K]$.

All that implies that the following two statements are equivalent in $M[K]$ :

(*) For every $f: j(\theta) \rightarrow \omega$ there are $\gamma<\delta$ in $A$ such that $(f(\gamma), f(\delta)) \in$ $H_{\gamma \delta}^{\prime}$.

(**) For every $f: \theta \rightarrow \omega$ there are $\alpha<\beta<\theta$ such that $(f(\alpha), f(\beta)) \in$ $H_{\alpha \beta}$.

As was metioned before, (b) holds for $\mathscr{H}$ in $M[G]$ and $M[G] \models " j(\mathbb{P})^{\kappa}$ is an $\omega_{1}$-closed partial order". Therefore, by the main lemma, $(* *)$ (and hence $(*)$ ) holds in $M[K]$.

We conclude that in $M[K]$, there is $A \in[j(\theta)]^{<j(\kappa)}$ such that for every $f: j(\theta) \rightarrow \omega$ there are $\gamma<\delta$ in $A$ with $(f(\gamma), f(\delta)) \in j^{*}(\mathscr{H})_{\gamma \delta} \quad\left(=H_{\gamma \delta}^{\prime}\right)$.

Therefore, by elementarity of $j^{*}$, in $V[G]$ there is an $A \in[\theta] \leq \aleph_{1}$ such that for every $f: \theta \rightarrow \omega$ there are $\alpha<\beta$ in $A$ with $(f(\alpha), f(\beta)) \in H_{\alpha \beta}$.

We can also prove Theorems 4 and 5 replacing the Levy collapse by the Mitchell collapse [Mi, DJW] and thus obtain the conclusions of these theorems in models where $\mathrm{CH}$ fails.

From the existence of a huge cardinal, Tall [Ta2] obtains the consistency of "first countable weakly $\aleph_{1}$-cwH spaces are weakly $\aleph_{2}$-cwH". He uses an even stronger axiom of infinity to obtain the consistency of "first countable weakly $\aleph_{1}$-cwH spaces are weakly cwH". So we use much weaker hypotheses to obtain slightly weaker results.

\section{INTEGER-VALUED FUNCTIONS}

As was mentioned before, Todorcevic proved that $\square(\theta)$ implies that there is a $\theta$-good subset of $F_{\theta, \omega} \times F_{\theta, \omega}$ [To2]. In [DW], it is proved that all one needs is a nonextendible monotone $\theta$-family of functions (which exists under $\square(\theta)$ but not in the model of Theorem 5).

Let $g, h$ be functions with range a subset of $\omega$. We say that $g$ weakly bounds $h$ if there is $n \in \omega$ such that for every $x \in \operatorname{dom}(g) \cap \operatorname{dom}(h)$

$$
g(x)+n>h(x) \quad \text { (in short, } g+n>h) \text {. }
$$

We say that $\mathscr{H}=\left\{h_{\beta}: \beta<\theta\right\}$ is a $\theta$-family of functions if the domain of $h_{\beta}$ is $\beta$. The $\theta$-family $\mathscr{H}$ is weakly bounded if there is a $g$ that weakly bounds each element of $\mathscr{H}$. We say that $\mathscr{H}$ is an initially weakly bounded (i.w.b.) $\theta$-family if for every $A \in[\theta]^{<\theta},\left\{h_{\beta}: \beta \in A\right\}$ is weakly bounded. The family is nonextendible if $\left\{h_{\beta}: \beta<\theta\right\}$ is not weakly bounded.

We can now repeat the construction of a $\theta$-good subset of $F_{\theta, \omega} \times F_{\theta, \omega}$ from a nonextendible i.w.b. $\theta$-family $\left\{h_{\beta}: \beta<\theta\right\}$, using i.w.b. in place of monotonicity. To see this, define for every $\alpha<\beta<\theta$ the finite set $H_{\alpha \beta}=$ $\left\{(n, m) \in \omega \times \omega: n+m \leq h_{\beta}(\alpha)\right\}$ and check that $\mathscr{H}=\left\{H_{\alpha \beta}: \alpha<\beta<\theta\right\}$ satisfies (3) of Theorem 1. 
Question 3. Does the existence of a $\theta$-good subset of $F_{\theta, \omega} \times F_{\theta, \omega}$ imply the existence of a nonextendible i.w.b. $\theta$-family of functions?

Fleissner [F] used the combinatorial principle $E_{\theta}^{\omega}$ to construct a locally countable, locally compact Moore space that is $<\theta$-cwH but not $\leq \theta$-cwH. In particular, by [GT] (see (1) existence of a $\theta$-good subset of $F_{\theta, \omega} \times F_{\theta, \omega}$.

Theorem 6. Let $\theta$ be an uncountable regular cardinal, and let $E \subset\{\alpha \in \theta$ : $\operatorname{co} f(\alpha)=\omega\}$ be a nonreflecting stationary set. Then there is a $\theta$-family $\left\{h_{\beta}\right.$ : $\beta<\theta\}$ such that for every $A \subset \theta,\left\{h_{\beta}: \beta \in A\right\}$ is weakly bounded if and only if $A \cap E$ is nonstationary. (In particular, $\left\{h_{\beta}: \beta<\theta\right\}$ is a nonextendible i.w.b. $\theta$-family.)

Proof. For every $\alpha \in E$, fix $\left\{a_{\alpha}(n): n \in \omega\right\}$ an increasing $\omega$-sequence unbounded in $\alpha$. For every $\alpha<\beta<\theta$ define

$$
h_{\beta}(\alpha)= \begin{cases}\min \left\{n \in \omega: a_{\alpha}(n) \neq a_{\beta}(n)\right\} & \text { if }(\alpha \in E) \wedge(\beta \in E), \\ 0 & \text { otherwise. }\end{cases}
$$

It is enough to consider $A \subset E$. Let $A$ be stationary, and assume, by way of contradiction, that $g: \theta \rightarrow \omega$ is a weak bound for $\left\{h_{\beta}: \beta \in A\right\}$. There exists a stationary $B \subset A$ and $n \in \omega$ such that for every $\beta \in B, g+n>h_{\beta}$. Let $f=g+n$; then for every $\beta \in B, f>h_{\beta}$.

Now, there exists a stationary $S \subset B$, and $m \in \omega$ such that for every $\alpha, \beta \in S, f(\alpha)=m$ and $\left\langle a_{\alpha}(0), \ldots, a_{\alpha}(m)\right\rangle=\left\langle a_{\beta}(0), \ldots, a_{\beta}(m)\right\rangle$. Let $\alpha<\beta$ in $S$. Let $k=\min \left\{n \in \omega: a_{\alpha}(n) \neq a_{\beta}(n)\right\}$. Then $k>m$; therefore, $f(\alpha)=m<k=h_{\beta}(\alpha)$, a contradiction.

Next we prove, by induction on $\gamma<\theta$, that there exists $g_{\gamma}: \gamma \rightarrow \omega$ that weakly bounds $\left\{h_{\beta}: \beta<\gamma\right\}$.

The successor case is easy, so let us assume that $\gamma$ is a limit. Let $C \subset \gamma$ be a club with $C \cap E \cap \gamma=\varnothing$. For every $\beta \in E \cap \gamma$ let

$$
\gamma^{+}(\beta)=\min (C \backslash \beta), \quad \gamma^{-}(\beta)=\max (C \cap \beta) .
$$

Notice that for every $\beta \in E \cap \gamma$ we have $\gamma^{-}(\beta)<\beta<\gamma^{+}(\beta)<\gamma$. Now define

$$
g_{\gamma}(\beta)= \begin{cases}g_{\gamma^{+}(\beta)}(\beta) & \text { if } \beta \in E, \\ 1 & \text { otherwise. }\end{cases}
$$

Let us show that $g_{\gamma}$ weakly bounds $\left\{h_{\beta}: \beta<\gamma\right\}$. If $\beta \notin E$, then $h_{\beta}$ is identically zero, so it suffices to consider $\beta \in E \cap \gamma$. Let $n=k+m+1$, where $k$ is the least integer such that $a_{\beta}(k)>\gamma^{-}(\beta)$ and $m$ is satisfies $g_{\gamma^{+}(\beta)}+m>h_{\beta}$. Let us show that $g_{\gamma}+n>h_{\beta}$. Let $\alpha \in E \cap \beta$.

Case 1: $\gamma^{-}(\alpha)=\gamma^{-}(\beta)$. In this case, $\gamma^{+}(\alpha)=\gamma^{+}(\beta)$; therefore $g_{\gamma}(\alpha)=$ $g_{\gamma^{+}(\alpha)}(\alpha)=g_{\gamma^{+}(\beta)}(\alpha)$. But $g_{\gamma^{+}(\beta)}(\alpha)+m>h_{\beta}(\alpha)$; therefore, $g_{\gamma}(\alpha)+n>h_{\beta}(\alpha)$.

Case 2: $\alpha<\gamma^{-}(\beta)$. Then $k+1>h_{\beta}(\alpha)$ because $a_{\beta}(k)>\gamma^{-}(\beta)$; therefore, $g_{\gamma}(\alpha)+n>h_{\beta}(\alpha)$.

Finally, let $A \subset E$ be nonstationary. Let us use $\left\{g_{\gamma}: \gamma<\theta\right\}$ to produce $g: \theta \rightarrow \omega$ that weakly bounds $\left\{h_{\beta}: \beta \in A\right\}$. Let $C \subset \theta$ be a club such that $C \cap A=\varnothing$. For every $\beta \in E$ let

$$
\delta^{+}(\beta)=\min (C \backslash(\beta+1)), \quad \delta^{-}(\beta)=\sup (C \cap \beta),
$$




$$
g(\beta)= \begin{cases}g_{\delta+(\beta)}(\beta) & \text { if } \beta \in E, \\ 1 & \text { otherwise. }\end{cases}
$$

Let $\beta \in A$. As before, let $n=k+m+1$, where $k$ is the least such that $a_{\beta}(k)>\delta^{-}(\beta)$ (notice, $\beta \in A$ implies that $\delta^{-}(\beta)<\beta$ ) and $m$ is such that $g_{\delta+(\beta)}+m>h_{\beta}$. Let us show that $g+n>h_{\beta}$. Let $\alpha \in E \cap \beta$.

Case 1: $\delta^{-}(\alpha)=\delta^{-}(\beta)$. So, $\delta^{+}(\alpha)=\delta^{+}(\beta)$; therefore, $g(\alpha)=g_{\delta^{+}(\alpha)}(\alpha)=$ $g_{\delta^{+}(\beta)}(\alpha)$. But $g_{\delta^{+}(\beta)}(\alpha)+m>h_{\beta}(\alpha)$; therefore, $g(\alpha)+n>h_{\beta}(\alpha)$.

Case 2: $\delta^{-}(\alpha)<\delta^{-}(\beta)$. In this case $\delta^{-}(\beta) \geq \alpha$. But $a_{\beta}(k)>\delta^{-}(\beta)$; therefore, $a_{\beta}(k)>\alpha$ and hence $a_{\beta}(k) \neq a_{\alpha}(k)$. So, $k+1>h_{\beta}(\alpha)$; therefore, $g(\alpha)+n>h_{\beta}(\alpha)$.

We remark here that $\neg E_{\omega_{2}}^{\omega}$ is equiconsistent with a Mahlo cardinal [D, HS], while $\neg \square\left(\omega_{2}\right)$ is equiconsistent with a weakly compact cardinal [Ma, To1, Be] (and therefore $\square\left(\omega_{2}\right)$ does not imply $\left.E_{\omega_{2}}^{\omega}\right)$. So consistency-wise, a result from $\square\left(\omega_{2}\right)$ is better (more difficult to deny) than one from $E_{\omega_{2}}^{\omega}$. On the other hand, $E_{\omega_{2}}^{\omega}$ does not imply $\square\left(\omega_{2}\right)$ (PFA is consistent with $E_{\omega_{2}}^{\omega}$ but implies $\neg \square\left(\omega_{2}\right)$ [Be]).

Theorem 7. Let $\kappa$ be a supercompact cardinal, let $\mathbb{P}$ be the Levy collapse of $\kappa$ to $\omega_{2}$ with countable conditions, and let $G$ be a P-generic filter over $V$. Then in $V[G]$ the following holds: For every regular $\theta \geq \omega_{2}$ and every $\theta$ family $\left\{h_{\beta}: \beta<\theta\right\}$, if for all $A \in[\theta]^{\aleph_{1}},\left\{h_{\beta}: \beta \in A\right\}$ is weakly bounded, then for every stationary set $S \subset \theta$ there exists a stationary $T \subset S$ such that $\left\{h_{\beta}: \beta \in T\right\}$ is weakly bounded.

The proof of Theorem 7 is very much like the proof of Theorem 5. The following lemma is the analog of the Main Lemma; the rest of the proof of the theorem is left to the reader.

Lemma. Assume that $\mathbb{P}$ is an $\omega_{1}$-closed partial order and that $\theta \geq \omega_{2}$ is a regular cardinal. Assume that $\mathscr{H}=\left\{h_{\beta}: \beta<\theta\right\}$ is a $\theta$-family and $S \subset \theta$ is stationary such that for every stationary $T \subset S$ the family $\left\{h_{\beta}: \beta \in T\right\}$ is not weakly bounded. Assume that $G$ is a $\mathbb{P}$-generic filter over $V$. Then in $V[G]$, $\left\{h_{\beta}: \beta \in S\right\}$ is not weakly bounded.

Proof. We proceed as in our Main Lemma and assume the lemma is false. Let $p_{0} \in \mathbb{P}$ and $\dot{g}$, a $\mathbb{P}$-name, be such that $p_{0} \Vdash$ " $\dot{g}: \theta \rightarrow \omega$ weakly bounds $\left\{h_{\beta}: \beta \in S\right\}$ ".

Let $M$ be an elementary substructure of $H(\lambda)$ for some large enough regular $\lambda$, with $S, \theta, \mathscr{H}, \mathbb{P}, p_{0}, \dot{g}$, and $\vdash_{\mathbf{P}}$ all members of $M$ and $M \cap \theta=\beta \in S$.

Construct $p_{0} \geq p_{1} \geq \cdots \geq p_{n+1} \geq \cdots$ such that $p_{n+1} \in M$ and $\alpha_{n}<\beta$ with

$$
p_{n+1} \Vdash " \dot{g}\left(\alpha_{n}\right)+n \leq h_{\beta}\left(\alpha_{n}\right) " .
$$

This process must stop at a finite stage since otherwise one could choose $q$ with $q \leq p_{n}$ for every $n \in \omega$ and get that for every $n \in \omega$ there is $\alpha_{n}<\beta$ such that $q \Vdash$ " $\dot{g}\left(\alpha_{n}\right)+n \leq h_{\beta}\left(\alpha_{n}\right)$ ", which is impossible.

So, there is $n \in \omega$ such that for every $q \leq p_{n}$ and every $\alpha<\beta$, if $q \in M$, then $q \nVdash$ “ $\dot{g}(\alpha)+\bar{n} \leq h_{\beta}(\alpha)$ ". As in the Main Lemma, it follows that for every $\alpha<\beta$,

$$
p_{n} \Vdash “ \dot{g}(\alpha)+\bar{n}>h_{\beta}(\alpha) ” .
$$


Let $T=\left\{\beta \in S:(\forall \alpha<\beta) p_{n} \Vdash\right.$ “ $\dot{g}(\alpha)+\bar{n}>h_{\beta}(\alpha)$ ” $\}$. By the elementarity of $M, T$ is stationary.

Now, for every $\alpha \in \theta$, let $q_{\alpha} \leq p_{n}$ and $m_{\alpha} \in \omega$ be such that $q_{\alpha} \Vdash$ " $\dot{g}(\alpha)=$ $m_{\alpha}$ ". In $V$, define $f: \theta \rightarrow \omega$ by $f(\alpha)=m_{\alpha}+\bar{n}$. Finally, continue as in the Main Lemma to get a contradiction by showing that for every $\beta \in T$ and every $\alpha<\beta, h_{\beta}(\alpha)<f(\alpha)$. In particular, $f$ weakly bounds $\left\{h_{\beta}: \beta \in T\right\}$.

By Theorem 6, in the model of Theorem $7, E_{\theta}^{\omega}$ fails for every regular $\theta \geq \omega_{2}$ (this is a result of Shelah). As was the case for Theorems 4 and 5, Theorem 7 can also be proved for the Mitchell collapse instead of the Levy collapse. We finish with a question.

Question 4. Is it consistent (relative to a large cardinal) that every i.w.b. $\omega_{2-}$ family extends?

\section{REFERENCES}

[Ba] J. Baumgartner, A new class of order types, Ann. Math. Logic 9 (1976), 187-222.

[Be] M. Bekkali, Topics in set theory, Springer-Verlag, New York, 1991.

[D] P. Daniels, A first countable, weakly $\omega_{1}-C W H$, not weakly $\omega_{2}-C W H$ space, Questions Answers Gen. Topology 2 (1988), 129-134.

[De] K. J. Devlin, Aspects of constructibility, Lecture Notes in Math., vol. 354, Springer-Verlag, New York, 1973.

[DJW] A. Dow, I. Juhasz, and W. Weiss, Integer-valued functions and increasing unions of first countable spaces, Israel J. Math. 67 (1989), 181-192.

[Do] A. Dow, Set-theory in topology, preprint, 1992.

[DTW1] A. Dow, F. Tall, and W. Weiss, New proofs of the consistency of the normal Moore space conjecture. I, Topology Appl. 37 (1990), 33-51.

[DTW2] _ New proofs of the consistency of the normal Moore space conjecture. II, Topology Appl. 37 (1990), 115-129.

[DW] A. Dow and S. Watson, A subcategory of TOP, preprint.

[F] W. G. Fleissner, On A-collectionwise Hausdorff spaces, Topology Proc. 2 (1977), 445-456.

[FS] W. G. Fleissner and S. Shelah, Collectionwise Hausdorff: Incompactness at singulars, Topology Appl. 31 (1989), 101-107.

[GT] G. Gruenhage and Tamano, handwritten notes.

[HS] L. Harrington and S. Shelah, Some exact equiconsistency results in set theory, Notre Dame J. Formal Logic 26 (1985), 178-188.

[J] T. Jech, Set theory, Academic Press, New York, 1978.

[KM] A. Kanamori and M. Magidor, The evolution of large cardinal axioms in set theory, Higher Set Theory, Lecture Notes in Math., vol. 699, Springer-Verlag, New York, 1978, pp. 99-275.

[K] K. Kunen, Set theory, North-Holland, Amsterdam, 1980.

[Ma] M. Magidor, Reflecting stationary sets, J. Symbolic Logic 47 (1982), 755-771.

[Mi] W. Mitchell, Aronszajn trees and the independence of the transfer property, Ann. Math. Logic 5 (1972), 21-46.

[S] S. Shelah, Remarks on $\lambda$-collectionwise hausdorff spaces, Topology Proc. 2 (1977), 583-592.

[Ta1] F. D. Tall, Weakly collectionwise Hausdorff spaces, Topology Proc. 1 (1976), 295-304.

[Ta2] , Topological applications of generic huge embeddings, Trans. Amer. Math. Soc. 341 (1994), 45-68. 
[To1] S. Todorcevic, Partitioning pairs of countable ordinals, Acta Math. 159 (1987), 261-294. [To2] — My new fan, handwritten notes, 1989.

[W] S. Watson, Comments on separation, Topology Proc. 14 (1989), 315-372.

Department of Mathematics, The University of Kansas, LaWrence, Kansas 66045

Current address, T. Laberge: Department of Mathematics, Union College, Schenectady, New York 12308

E-mail address: labergetegar.union.edu

Current address, A. Landver: IBM Israel-Science \& Technology, MATAM, Haifa 31905, Israel

E-mail address: landver@haifasc3. vnet.ibm.com 